\title{
Thermoanalytische Untersuchungen zur Rückoxidation von $\mathrm{Ba}_{2} \mathrm{YCu}_{3} \mathrm{O}_{6+x}$
}

\author{
Chr. L. Teske und Hk. Müller-Buschbaum \\ Institut für Anorganische Chemie der Christian-Albrechts-Universität Kiel, D-2300 Kiel, FRG
}

Z. Naturforsch. 43a, 965-970 (1988); eingegangen am 8. August 1988

Thermoanalytical Investigations about the Reoxidation of $\mathrm{Ba}_{2} \mathrm{YCu}_{3} \mathrm{O}_{6+x}$

The reoxidation reaction of $\mathrm{Ba}_{2} \mathrm{YCu}_{3} \mathrm{O}_{6+x}$ forming the superconducting ceramic material $\mathrm{Ba}_{2} \mathrm{YCu}_{3} \mathrm{O}_{6.93}$ is studied by thermoanalytical methods (DTA and TG). The exothermal oxygen uptake in pure $\mathrm{O}_{2}$-atmosphere is at a maximum rate in the range of $230-330^{\circ} \mathrm{C}(\approx 150 \mathrm{~min})$. The reoxidation temperature can be lowered by treatment of $\mathrm{Ba}_{2} \mathrm{YCu}_{3} \mathrm{O}_{6.0}$ in $\mathrm{Ar}: \mathrm{H}_{2}=1: 1$ atmosphere but the uptake of greater amounts of hydrogen prevents the material from becoming super-conducting again at low reoxidation temperatures. The process has an activation energy $E_{a}=70.9 \pm 2 \mathrm{~kJ} / \mathrm{Mol}$ and a reaction enthalpy $\Delta H=-86.7 \mathrm{~kJ} / \mathrm{Mol} \mathrm{Ba}_{2} \mathrm{YCu}_{3} \mathrm{O}_{6.0}$ (normalized to a $1 / 2 \mathrm{O}_{2}$ basis) which is estimated from DTA-data.

Keywords: $\mathrm{Ba}_{2} \mathrm{YCu}_{3} \mathrm{O}_{6+x}$, Thermoanalysis, Reoxidation, Activation Energy, Reaction Enthalpy.

\section{Einleitung}

Seit der Entdeckung supraleitender Oxocuprate $[1,2]$ erschienen viele Arbeiten über Synthese, Struktur und supraleitende Eigenschaften dieser Stoffklasse. Für den Chemiker ist die Bildung der supraleitenden Phase $\mathrm{Ba}_{2} \mathrm{YCu}_{3} \mathrm{O}_{6+x}$ (mit $x \approx 1,0$ ) aus der nichtsupraleitenden Substanz $\mathrm{Ba}_{2} \mathrm{YCu}_{3} \mathrm{O}_{6,0}$ von besonderem Interesse. Auch hierzu gibt es schon Untersuchungen. Beschränkt man diese auf thermoanalytische Arbeiten, so ist festzustellen, daß eine Reihe von Autoren den Abbau und Aufbau des Supraleiters $\mathrm{Ba}_{2} \mathrm{YCu}_{3} \mathrm{O}_{6+x}$ mit dieser Arbeitstechnik studierten [3-12]. Prinzipielle thermoanalytische Abbau- und Aufbauuntersuchungen in Abhängigkeit von der Temperatur seien hervorgehoben [9,13]. Andere Autoren befassen sich mit dem Bereich höherer Temperaturen von etwa $500^{\circ} \mathrm{C}$ bis maximal $1200^{\circ} \mathrm{C}[6,8]$. In der Regel werden thermische Zersetzung und Reorganisation des supraleitenden Kristallgitters durch Abbau des Supraleiters unter Vakuum oder Inertgas und Aufbau an Luft bzw. Sauerstoff studiert [3, 4, 7, 10, 11, 12]. Besonderes Gewicht liegt auch auf dem Phasenwechsel orthorhombisch $\leftrightarrow$ tetragonal in Verbindung mit dem Sauerstoffgehalt des supraleitenden Kristallgitters [5].

Sonderdruckanforderungen an Prof. Dr. Hk. Müller-Buschbaum oder Dr. Chr. L. Teske, Institut für Anorganische Chemie der Christian-Albrechts-Universität, Olshausenstraße 40, Otto-Hahn-Platz 6/7, D-2300 Kiel 1.
Bei der Mehrzahl aller bisher erschienenen Arbeiten fällt auf, daß die Heizraten der thermoanalytischen Experimente relativ hoch sind $\left(>10^{\circ} / \mathrm{min}\right)$ und $1^{\circ} / \mathrm{min}$ nicht unterschreiten. Da die Reorganisation des supraleitenden Kristallgitters ein diffusionskontrollierter Vorgang ist, zeigten eigene Experimente, daß die Rückoxidationstemperaturen von der Heizrate stark verfälscht werden können. Hier wird über die Reorganisation des $\mathrm{Ba}_{2} \mathrm{YCu}_{3} \mathrm{O}_{6,93}$-Supraleiters bei besonders kleinen Aufheizraten berichtet. Die Aktivierungsenergie der Rückoxidation und die Rückoxidationsenthalpie wurden bestimmt und eine Erklärung gefunden, weshalb nach Kissinger's Methode [14] gemessene Aktivierungsenergien hier nicht kritiklos übernommen werden können.

\section{Experimenteller Teil}

\section{Darstellung des Supraleiters $\mathrm{Ba}_{2} \mathrm{YCu}_{3} \mathrm{O}_{6,93}$}

Für die thermoanalytischen Experimente wurde zunächst supraleitendes Material $\mathrm{Ba}_{2} \mathrm{YCu}_{3} \mathrm{O}_{6,93}$ präpariert. Hierzu wurde die Mischung $\mathrm{Ba}(\mathrm{OH})_{2} \cdot 8 \mathrm{H}_{2} \mathrm{O}$ : $\mathrm{Y}_{2} \mathrm{O}_{3}: \mathrm{CuO}=2: 0,5: 3$ innig vermengt und bei $150-450^{\circ} \mathrm{C}$ der überwiegende Teil des Kristallwassers von $\mathrm{Ba}(\mathrm{OH})_{2} \cdot 8 \mathrm{H}_{2} \mathrm{O}$ abgebaut. Anschließend wurde in einer Kugelmühle vermahlen, zu Tabletten $(30 \mathrm{t}$, $23 \mathrm{~mm} \varnothing$ ) verpreßt und bei $950^{\circ} \mathrm{C}, 12 \mathrm{~h}$ erhitzt. Nach dem Abkühlen wurde die gesinterte Tablette ca. $5 \mathrm{~h}$ zwischen $400-430^{\circ} \mathrm{C}$ an Luft getempert. Die so er- 
haltenen Präparate haben ein $T_{\mathrm{c}}$ von $90-91 \mathrm{~K}$ (s. Abb. 2, A). Typische Gitterkonstanten (in $\AA$ ) solcher Präparate sind: $a=3,8213 \pm 0,0005, b=3,8862$ $\pm 0,0007, c=11,6809 \pm 0,0010$. Verwiesen sei hier auf eine Untersuchung der Abhängigkeit der Zellparameter vom Sauerstoffgehalt [15]. Die aufgeführten Werte sind mit der Zusammensetzung $\mathrm{Ba}_{2} \mathrm{YCu}_{3} \mathrm{O}_{6,93}$ vereinbar.

\section{Thermischer Abbau und Rückoxidation}

Vor jedem thermoanalytischen Experiment zur Rückoxidation wird die supraleitende Phase $\mathrm{Ba}_{2} \mathrm{YCu}_{3} \mathrm{O}_{6.93}$ in Argonatmosphäre bis auf $950^{\circ} \mathrm{C}$ aufgeheizt. Wie TG-Messungen zeigen, verliert kompaktes Material zwischen 300 und $950^{\circ} \mathrm{C}$ Sauerstoff $(2,3 \%)$. Abbildung $1 \mathrm{~A}$ gibt den Verlauf von TG-Kurven wieder; man erkennt, daß bei wiederholtem Abbau keine signifikanten Unterschiede auftreten.

Die Rückoxidationsexperimente gehen von thermisch abgebautem Material der Zusammensetzung $\mathrm{Ba}_{2} \mathrm{YCu}_{3} \mathrm{O}_{6,0}$ aus. Rückoxydiert wurde in reiner Sauerstoffatmosphäre. Abbildung $1 \mathrm{~B}$ zeigt mit den Kurven 4 und 5 die prozentuale Gewichtszunahme in Abhängigkeit von der Temperatur bei einer geringen Aufheizrate von $0,2^{\circ} / \mathrm{min}$. Kurve 5 stellt die Wiederholung der Messung nach Kurve 4 dar. Zwischen beiden Rückoxidationsmessungen liegt ein thermischer Abbau unter Argon, wie oben beschrieben. Man erkennt, daß eine Wiederholung der Rückoxidation am gleichen Präparat die Rückoxidationstemperatur zu tieferen Werten verschiebt. Weitere Zyklen dieser Art verbessern die Rückoxidationstemperatur dann nicht mehr. Die Kurven 6 und 7 (Abb. 1B) wurden nach
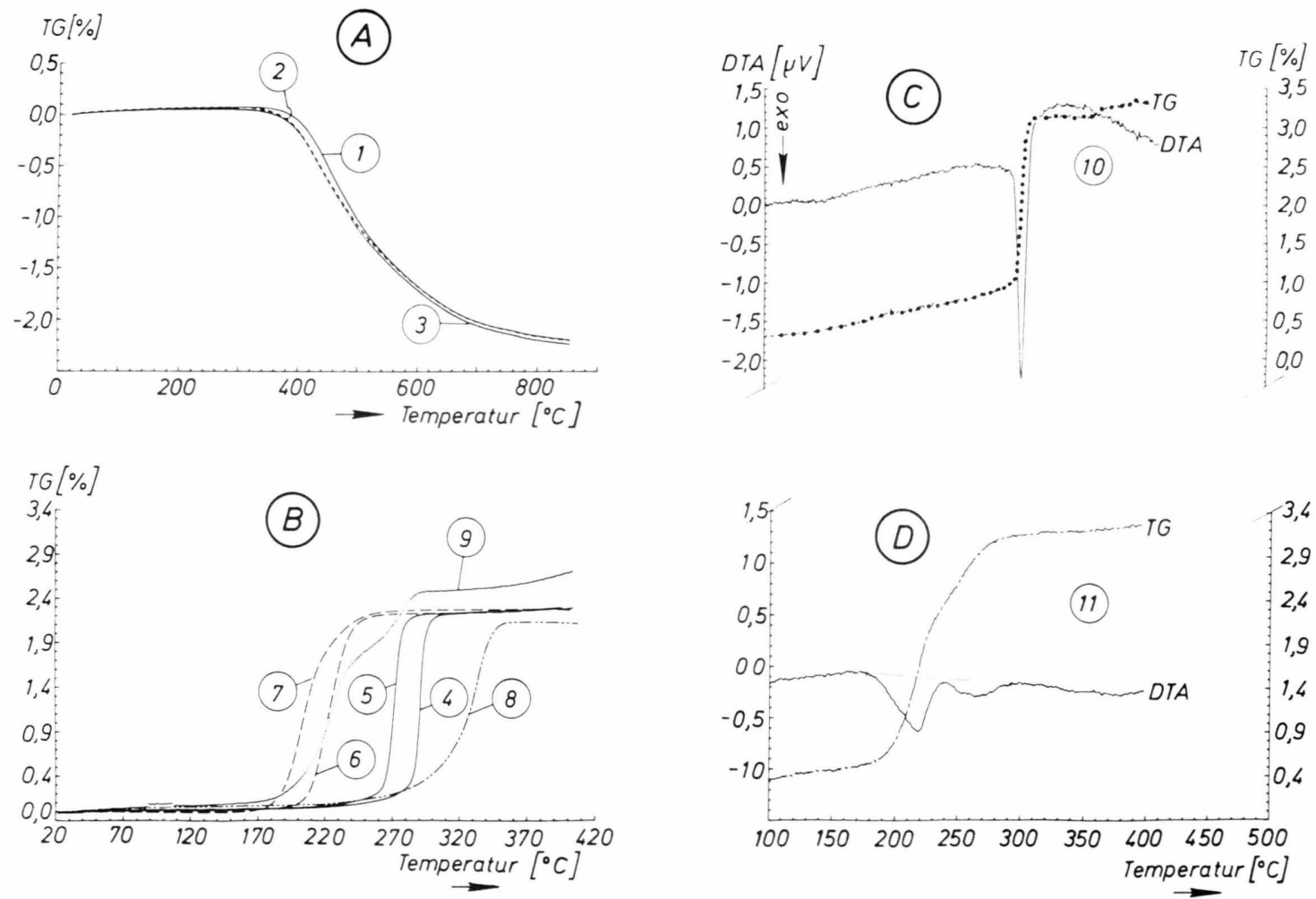

Abb. 1 A. Thermischer Abbau von $\mathrm{Ba}_{2} \mathrm{YCu}_{3} \mathrm{O}_{6.93}$ in Argonatmosphäre (Heizrate $1^{\circ}$ min). Kurven 2 u. 3 zweimalige Wiederholung mit derselben Probe (Gewichtsverlust 2.3\%). B) TG-Messungen zur Rückoxidation von $\mathrm{Ba}_{2} \mathrm{YCu}_{3} \mathrm{O}_{6} .0$ ( $\mathrm{Heizrate}$ $0,2 / \mathrm{min}$ ). Kurven 4 u. 5 nach Abbau in Argon. Kurven 6 u. 7 nach Behandlung mit Ar: $\mathrm{H}_{2}=1: 1$. Kurve 9 nach „Langzeitbehandlung" in Ar: $\mathrm{H}_{2}=1: 1$ (s. Text). Kurve $8=$ pulverförmiges Material. Kurven $5-7$ u. $9=$ kompaktes Material. C) Simultane DTA/TG-Messung zur Rückoxidation von $\mathrm{Ba}_{2} \mathrm{YCu}_{3} \mathrm{O}_{6.0}$ in $\mathrm{O}_{2}$-Atmosphäre (Heizrate $0,3^{\circ} / \mathrm{min}$ ). Kompaktes Material, Ref. $\mathrm{Al}_{2} \mathrm{O}_{3}$, Gewichtszunahme 2.3\%. D) Simultane DTA TG-Messung in $\mathrm{O}_{2}$-Atmosphäre. Wiederholung des Versuchs von Kurve 10, aber nach „Langzeitbehandlung“ in $\mathrm{Ar}: \mathrm{H}_{2}=1: 1$ (s. Text). Heizrate 0,3\% min, Gewichtszunahme TG $>2,5 \%$. 
einer zusätzlichen Behandlung in reduzierender Atmosphäre erhalten. Der unter Argon abgebaute Supraleiter wird in einer Mischung $\mathrm{Ar}: \mathrm{H}_{2}=1: 1 \mathrm{mit}$ $1^{\circ} /$ min bis auf $320^{\circ} \mathrm{C}$ aufgeheizt. Es folgt ein Abkühlvorgang mit $10^{\circ} / \mathrm{min}$ bis auf $200^{\circ} \mathrm{C}$. Innerhalb einer weiteren Stunde wird schließlich Raumtemperatur erreicht. Aus Kurve 6, Abb. 1 B, erkennt man, daß der Beginn der Rückoxidation des kompakten Materials um weitere $80^{\circ}$ zu tieferen Temperaturen verschoben ist. Eine Wiederholung ergibt auch unter diesen Bedingungen eine nochmalige Absenkung der Rückoxidationstemperatur. Wird der bis $320^{\circ} \mathrm{C}$ in $\mathrm{Ar}: \mathrm{H}_{2}=1: 1$ aufgeheizte Stoff über $2 \mathrm{~h}$ dieser reduzierenden Atmosphäre ausgesetzt, so zeigt er ein anderes Verhalten bei der Rückoxidation in $\mathrm{O}_{2}$ (siehe Kurve 9, Abbildung $1 \mathrm{~B})$. Man erkennt zwei Wendepunkte, die gesamte Sauerstoffaufnahme ist hier größer als 2,4\%. Hervorgehoben sei, daß alle Meßkurven (Abb. 1 B, Kurven 4-7 und 9) an gepreßtem kompakten Material erhalten wurden. Kurve 8 (Abb. 1 B) entspricht dem Präparat von Kurve 4, wurde jedoch an pulverförmigem Material aufgenommen. Erstaunlich ist die etwas geringere Sauerstoffaufnahme von 2,2\% und die langsamere Rückoxidation.

Abbildung 2 zeigt Beispiele von Messungen zur Widerstandsänderung mit der Temperatur*. Es ist zu erkennen, daß das supraleitende Material nach einer Langzeitbehandlung in reduzierender Atmosphäre $\left(\mathrm{Ar}: \mathrm{H}_{2}\right)$ bei der Rückoxidation bis $420^{\circ} \mathrm{C}$ nicht restauriert werden kann.

Rückoxidationen mit simultanen TG- und DTAMessungen gibt Abb. 1C und 1D wieder. Kurve 10 (Abb. 1C) zeigt, daß die Reorganisation des supraleitenden Kristallgitters von einem exothermen Effekt begleitet wird. Die Fläche unter der Kurve entspricht $-124 \mathrm{~J} / \mathrm{g}$. Bemerkenswert ist der Verlauf der DTAKurve eines in $\mathrm{Ar}: \mathrm{H}_{2}=1: 1$ langzeitbehandelten Präparats. Man erkennt, daß den beiden Wendepunkten der TG-Kurve je ein exothermer Peak der DTAKurve entspricht. In der Diskussion wird hierauf eingegangen.

\section{Bestimmung der Aktivierungsenergie und der Enthalpie der Rückoxidation}

Wie in der Einleitung erwähnt wurde, hängt die Rückoxidationstemperatur von der Heizrate ab. Dies

* Wir danken Herrn Prof. Rietschel, Kernforschungszentrum Karlsruhe und der Fa. Hoechst AG, Frankfurt, für die Ausführung der Leitfähigkeitsmessungen.
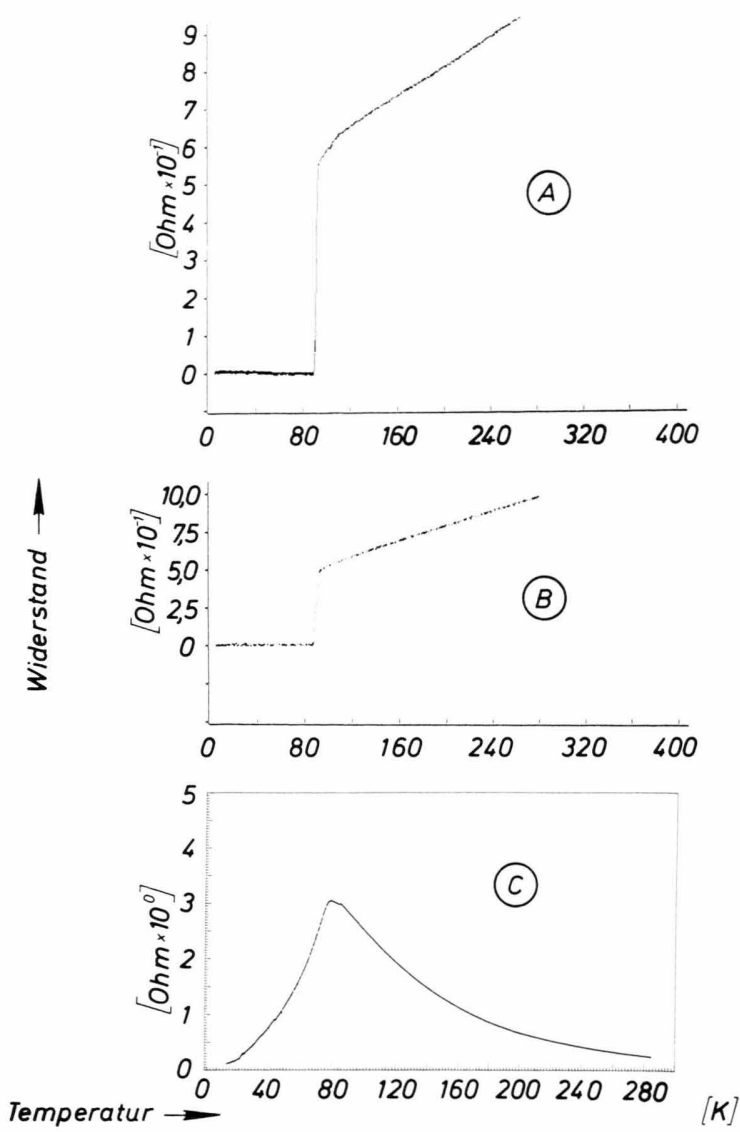

Abb. 2. Abhängigkeit des Widerstands von der Temperatur:

A) Messung nach Experiment 4 und 5 (Abb. 1 B);

B) Messung nach Experiment 6 und 7 (Abb. 1 B);

C) Messung nach Experiment 9 (Abb. 1 B).

demonstriert Abbildung $3 \mathrm{~A}$. Nach der Methode von Kissinger [14] kann man die Aktivierungsenergie für einfache Zersetzungsreaktionen unabhängig von der Reaktionsordnung durch Auswertung dieser Maxima bestimmen. Dies geschieht in Abb. 3 B aus der Steigung der Ausgleichsgeraden. Es ergibt sich eine Aktivierungsenergie der Rückoxidation von $E_{\mathrm{a}}=70,9$ $\pm 2 \mathrm{~kJ} / \mathrm{Mol}$.

Abbildung 4 zeigt drei DTA/TG-Messungen der Rückoxidation von $\mathrm{Ba}_{2} \mathrm{YCu}_{3} \mathrm{O}_{6,0}$ in $\mathrm{O}_{2}$ mit Basislinienkorrektur der DTA-Kurven. Trotz unterschiedlicher Heizraten sind die frei werdenden Enthalpien in vergleichbarer Größenordnung. Der mittlere Wert bei einer Sauerstoffaufnahme von 2,2\% (Pulverpräparate) beträgt $\Delta H=-120 \mathrm{~J} / \mathrm{g}$. Am kompakten Material (Kurve 10, Abb. 1 C) beträgt $\Delta H=-124 \mathrm{~J} / \mathrm{g}$ bei einer $\mathrm{O}_{2}$-Aufnahme von $2,3 \%$. Üblicherweise wird auf 

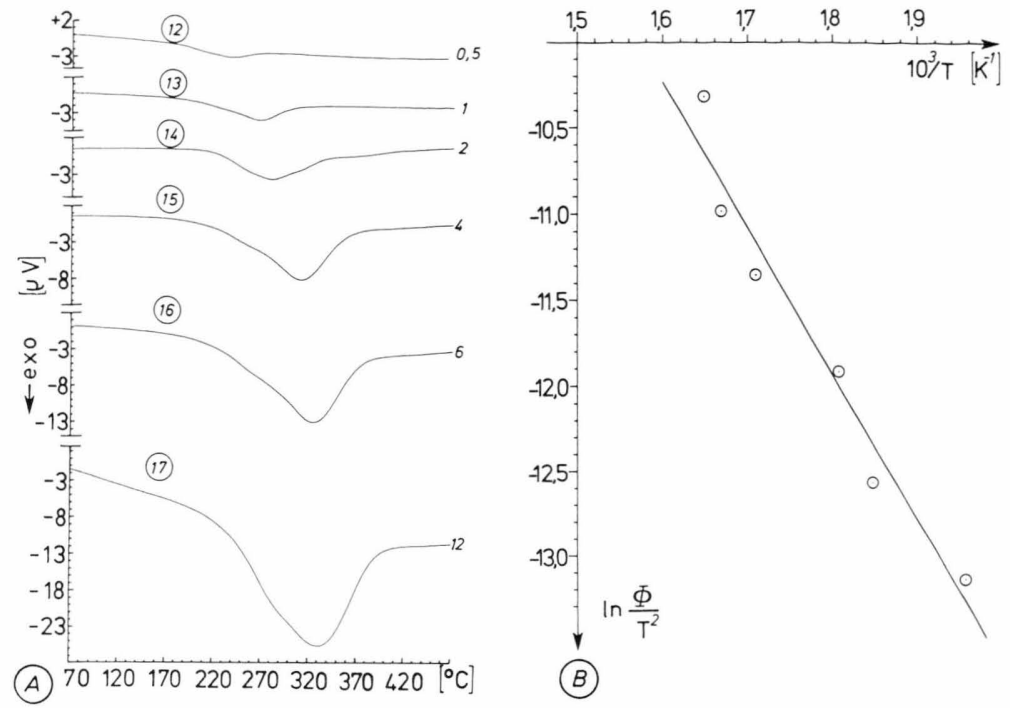

Abb. 3. A) DTA-Kurven für die Rückoxidation von $\mathrm{Ba}_{2} \mathrm{YCu}_{3} \mathrm{O}_{6,0}$ in $\mathrm{O}_{2}$-Atmosphäre bei verschiedenen Heizraten zwischen 0,5 und $12^{\circ} / \mathrm{min}$. Die Kurven $15-17$ entsprechen den DTA-Kurven von Abb. 4A mit verändertem Maßstab. B) Graphische Darstellung des Zusammenhangs zwischen der Heizrate $\Phi$ und der Lage $T[\mathrm{~K}]$ der DTA-Minima nach Kissinger [14]. Daten aus den Kurven 12-17.

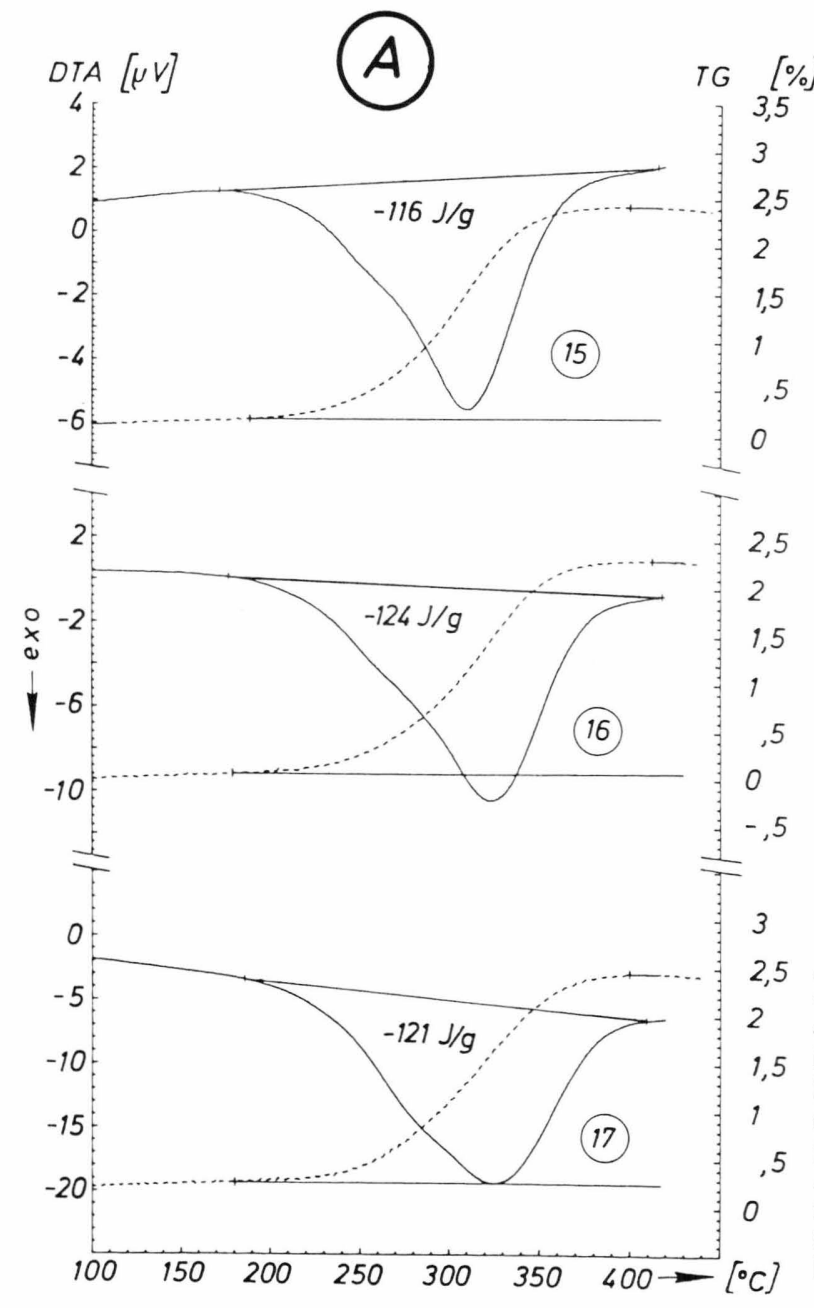

(B)

DTA $[\mu V]$

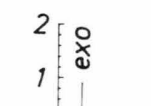

18

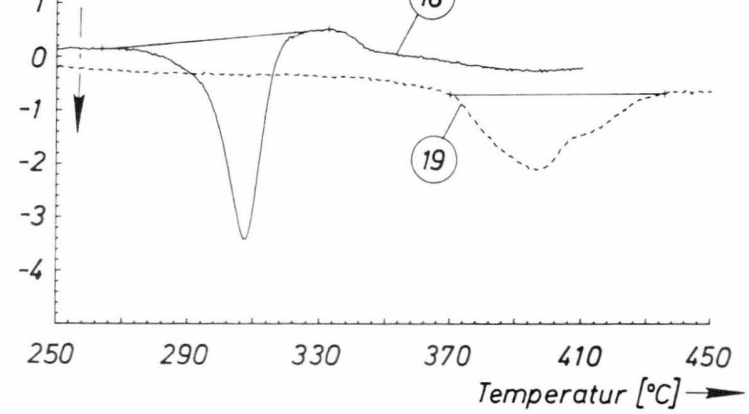

bb. 4. A) Simultane DTA/TG-Messung zur Rückoxidation von pulverförmigem $\mathrm{Ba}_{2} \mathrm{YCu}_{3} \mathrm{O}_{6,0}$ (Heizraten: Kurve 15, $4 \% \mathrm{~min}$; Kurve 16, $6 \% \mathrm{~min}$ u. Kurve 17, $12 \% \mathrm{~min}$. Ref. $\mathrm{Ba}_{2} \mathrm{YCu}_{3} \mathrm{O}_{6,93}$-Pulver. Mittlere Gewichtsänderung 2,2\%). Es wurde eine Basislinienkorrektur für die DTA-Kurven durchgeführt. B) DTA-Messung zur Rückoxidation an kompaktem $\mathrm{Ba}_{2} \mathrm{YCu}_{3} \mathrm{O}_{6}$ (Heizrate $0,3^{\circ} / \mathrm{min}$ ). Kurve 18 in $\mathrm{O}_{2}$ Atmosphäre. Kurve 19 Wiederholung des gleichen Experiments in getrockneter Luft. Die eingezeichneten Flächen unter den Kurven entsprechen jeweils den gleichen Energiebeträgen. 
eine Sauerstoffaufnahme von $0,5 \mathrm{O}_{2}$, entsprechend einer Massenänderung von 2,46\% normiert. Damit errechnet sich ein mittlerer Wert der Rückoxidationsenthalpie von $\Delta H=-133,4 \mathrm{~J} / \mathrm{g}=-86,7 \mathrm{~kJ} / \mathrm{Mol}$ $\mathrm{Ba}_{2} \mathrm{YCu}_{3} \mathrm{O}_{6,0}$.

\section{Diskussion der Ergebnisse}

Den TG-Messungen zur Rückoxidation von $\mathrm{Ba}_{2} \mathrm{YCu}_{3} \mathrm{O}_{6,0}$ in $\mathrm{O}_{2}$-Atmosphäre ist $\mathrm{zu}$ entnehmen, $\mathrm{da} ß$ die Reorganisation des supraleitenden Materials zwischen $260^{\circ}$ und $280^{\circ} \mathrm{C}$ beginnt und nach $150 \mathrm{~min}$ abgeschlossen ist. Andere Autoren [11,17] bestimmten mit $250-285^{\circ} \mathrm{C}$ ähnliche Werte. Eine kurzfristige Behandlung (siehe experimenteller Teil) von $\mathrm{Ba}_{2} \mathrm{YCu}_{3} \mathrm{O}_{6,0}$ mit $\mathrm{Ar}: \mathrm{H}_{2}=1: 1$ verschiebt den Beginn der Rückoxidation um maximal $80^{\circ}$ zu tieferen Temperaturen. Dies kann in der Praxis von Bedeutung sein, wenn $\mathrm{Ba}_{2} \mathrm{YCu}_{3} \mathrm{O}_{6,0}$ auf einem thermisch empfindlichen Träger rückoxidiert werden soll. Die Dauer der Rückoxidation verlängert sich hierdurch auf etwa $250 \mathrm{~min}$. Die Sauerstoffaufnahme hat für die unter Argon bzw. in Ar: $\mathrm{H}_{2}$-Atmosphäre erhaltenen Phasen den gleichen Wert von 2,3\%. Nach einer Langzeitbehandlung mit Ar: $\mathrm{H}_{2}=1: 1$ bei $320^{\circ} \mathrm{C}$ gelingt es nicht mehr, im mittleren Temperaturbereich den supraleitenden Zustand wiederherzustellen. Die Rückoxidation von Präparaten, die längere Zeit reduzierend behandelt wurden, verläuft in zwei Schritten (Kurve 9, Abbildung 1B). Wie simultane DTA/TG-Messungen zeigen (Abb. 1 D), erfolgt die Sauerstoffaufnahme in zwei exothermen Schritten. Der ab $180^{\circ} \mathrm{C}$ einsetzende exotherme Verlauf der DTA-Kurve kann mit der Umsetzung von eingelagertem Wasserstoff zu Wasser erklärt werden. Als gegenläufiger endothermer Effekt überlagert sich die Verdampfungsenthalpie des gebildeten Wassers. Der zweite, bei höheren Temperaturen beobachtbare DTA-Peak repräsentiert wahrscheinlich die Rückoxidation des Kupfers und die partielle Reorganisation des Kristallgitters, ohne die supraleitenden Eigenschaften zu erreichen. Schöllhorn und Mitarb. [16] fanden ebenfalls irreversible Strukturänderungen bei stark verlängerten Reaktionszeiten in $\mathrm{H}_{2}$-Atmosphäre. Für die praktische Anwendung ist eine kurze Behandlung mit Ar: $\mathrm{H}_{2}=1: 1$ günstig, da die Rückoxidation bei niedrigen Temperaturen beginnt und auch bei tieferen Temperaturen abgeschlossen ist, als bei Behandlung in reinem Argon.
Haller und Mitarb. [17] führten aus, daß die diffusionskontrollierte Sauerstoffaufnahme ein aktivierter Proze $B$ ist. Aus der Temperaturabhängigkeit der Sauerstoffaufnahme bestimmten sie die Aktivierungsenergie zu $E_{\mathrm{a}}=111 \mathrm{~kJ} / \mathrm{Mol}$. Dieser Wert entspricht der Größenordnung einer Leerstellendiffusion von Sauerstoff in Oxiden. Nach der hier angewandten Methode (Auswertung der heizratenabhängigen Lage der DTAMinima (Kissinger [14])) ergibt sich $E_{\mathrm{a}}=70,9 \mathrm{~kJ} / \mathrm{Mol}$. Für die relativ große Abweichung vom oben genannten Wert gibt es mehrere Erklärungen. Es kann ein prinzipieller Fehler der Methode nach Kissinger vorliegen, wenn z. B. das DTA-Minimum nicht mehr mit dem Maximum der Reaktionsgeschwindigkeit zusammenfällt. Es wurde jedoch auch beobachtet, daß $\mathrm{Ba}_{2} \mathrm{YCu}_{3} \mathrm{O}_{6,0}$ in $\mathrm{O}_{2}$ und in Luft ein unterschiedliches Rückoxidationsverhalten zeigt. Abbildung 4B zeigt den Verlauf der DTA-Kurve (Nr. 18) in reinem Sauerstoff im Vergleich zur Rückoxidation (Kurve 19) in Luft. Man erkennt, daß infolge des geringeren Sauerstoffpartialdrucks in Luft das DTA-Minimum beträchtlich zu höheren Temperaturen verschoben wird. Überträgt man diese Beobachtung auf die Messungen zur Bestimmung der Aktivierungsenergie der Rückoxidation von $\mathrm{Ba}_{2} \mathrm{YCu}_{3} \mathrm{O}_{6,0}$, so ist eine Verlagerung der DTA-Peaks zu höheren Temperaturen gleichbedeutend mit einem zu kleinen Wert $E_{\mathrm{a}}$. Abnehmender Sauerstoffpartialdruck als Folge der mit steigenden Heizraten zu höheren Temperaturen driftenden DTAPeaks verstärkt somit die Verschiebung der DTAPeaks zusätzlich zu höheren Temperaturen, d.h. die Steigung in Abb. 3 wird flacher.

Wie bereits oben erwähnt, ergibt sich bei den DTA-Experimenten die Reaktionsenthalpie für die Rückoxidation zu $\Delta H \approx-86,7 \mathrm{~kJ} / \mathrm{Mol}$. Nach P. K. Gallagher [3] liegt $\Delta H$ im Bereich zwischen $\Delta H=-24$ bis $-26,5 \mathrm{kcal} / \mathrm{Mol}$ entspr. $\Delta H=-100,4$ bis $-110,9 \mathrm{~kJ} / \mathrm{Mol}$ (normiert auf $1 / 2 \mathrm{O}_{2}$ ). Diese Werte werden zunächst aus Gleichgewichtsmessungen (log $\mathrm{PO}_{2}$ gegen $1 / T$; TG-Messungen) ermittelt. Die ergänzenden DSC-Messungen ergaben mit $\Delta H=15,5 \mathrm{kcal} / \mathrm{Mol}$ für den Abbau in $\mathrm{N}_{2}$-Atmosphäre bzw. $\Delta H=-28 \mathrm{kcal} / \mathrm{Mol}$ für die Rückoxidation in $\mathrm{O}_{2}$-Atmosphäre (entsprechend 64,9 bzw. $-117,2 \mathrm{~kJ} / \mathrm{Mol}$ ) einen wesentlich kleineren bzw. gröBeren Wert (Durchschnittsbetrag aus beiden Extrema: $91 \mathrm{~kJ} / \mathrm{Mol}$ ).

Eine Hauptschwierigkeit bei der Ermittlung dieser Daten aus den DSC- bzw. DTA-Experimenten liegt bei der Festlegung der Integrationsgrenzen, da sich 
$\triangle C p$ und somit auch die Basislinie im fraglichen Temperaturbereich stark ändern [3]. Wir haben diesen Einfluß bei unseren Messungen ebenfalls berücksichtigt und den Basislinienverlauf in entsprechenden Blindversuchen ermittelt (zum Verlauf der korrigierten Basislinie vgl. Abb. 4, Kurven 15, 16, 17, im Bereich $100-150^{\circ} \mathrm{C}$ ). Es muß dennoch eingeräumt werden, daß dieser Punkt die Meßgenauigkeit erheblich beeinflussen kann. Die DTA-Messungen enthalten weiterhin als unbekannte Größe die Umwandlungswärme tetragonal $\leftrightarrow$ orthorhombisch [3]. $\mathrm{Ba}_{2} \mathrm{YCu}_{3} \mathrm{O}_{6,0}$ entsteht beim Abbau des Supraleiters von $\mathrm{Ba}_{2} \mathrm{YCu}_{3} \mathrm{O}_{7-x}$ und kristallisiert tetragonal $(\mathrm{P} 4 / \mathrm{mmm})$, wie erst kürzlich nochmals auch mit Röntgeneinkristalldaten belegt wurde [18]. Dieser endotherme Betrag für die Umwandlungsenthalpie wird aber als vernachlässigbar klein eingeschätzt $[3,9]$, so daß er bei DTA- oder DSC-Experimenten bislang nicht erfaßt wurde.

[1] J. G. Bednorz u. K. A. Müller, Z. Phys. B 64, 189 (1986).

[2] M. K. Wu, J. R. Ashburn, C. J. Torng, P. H. Hor, R. L. Meng, L. Gao, Z J. Huang, Y. Q. Wang u. W. C. Chu, Phys. Rev. Lett. 58, 908 (1987).

[3] P. K. Gallagher, H. M. O’Bryan, S. A. Sunshine u. D. W. Murphy, Mat. Res. Bull. 22, 995 (1987).

[4] P. K. Gallagher, Adv. Ceram. Mater. 2, No. 3B, Spec. Iss. (1987).

[5] H. U. Krebs u. R. Wördenweber, J. Appl. Phys. 63(5), 1642 (1988).

[6] Z. Gabelica, E. G. Derouane, J. P. Vigneron, Ph. Lambin, M. Renier, A. A. Lucas, G. Deconnick, F. Bodart u. G. Demortier, Solid State Comm. 64, No. 9, 1221 (1987).

[7] Y. K. Huang, K. Kadowaki, M. Van Sprang, A. C. Moleman u. A. A. Mendovsky, J. Less, Common Metals 136, 169 (1987).

[8] L. P. Cook, C. K. Chiang, W. Wong-Ng u. J. Blendell, Adv. Ceram. Mater. 2, No. 3 B, Spec. Iss. (1987).

[9] G. Roth, D. Ewert, G. Heger, M. Hervieu, C. Michel, B. Raveau, F. D'Yvoire u. A. Revcolevschi, Z, Phys. B69, 21 (1987).
Zusammenfassend ist festzustellen, daß der thermische Abbau des supraleitenden Materials $\mathrm{Ba}_{2} \mathrm{YCu}_{3} \mathrm{O}_{7-x}$ in Ar-Atmosphäre unabhängig von der Zahl der Abbauzyklen im gleichen Temperaturbereich liegt. Der Temperaturbereich der Rückoxidation (Aufbau des supraleitenden Gitters) wird von der äuBeren Beschaffenheit (Pulver oder kompaktes Material), dem Sauerstoffpartialdruck und der Anzahl der Rückoxidationszyklen beeinflußt. Der Beginn der Rückoxidation verschiebt sich bei einer Behandlung in einer $\mathrm{Ar} / \mathrm{H}_{2}$-Atmosphäre zu deutlich tieferen Temperaturen, was für technische Anwendungen von Bedeutung sein kann.

Die Experimente wurden mit der Simultan Thermoanalysenapparatur STA 429 der Fa. Netzsch ausgeführt. Probenträger: 6.225.6-70+S (DTA) bzw. 6.225.6-57+S (TG).

[10] C. K. Chiang, L. P. Cook, S. S. Chang, J. E. Blendell u. R. S. Roth, Adv. Ceram. Mater. 2, No. 3 B, Spec. Iss. (1987).

[11] S. R. Dharwadkar, V. S. Jakkal, J. V. Yakhmi, I. K. Gopalakrishnan u. R. M. Iyer, Solid State Comm. 64, No. 12, 1429 (1987).

[12] E. Takayama-Muromachi, Y. Uchida, K. Yukino, T. Tanaka u. K. Kato, Japanese J. Appl. Phys. 26, No. 5, L665 (1987).

[13] T. Ozawa, A. Negishi, Y. Takahashi, R. Sakamoto u. H. Ihara, Thermochim. Acta 124, 147 (1988).

[14] H. E. Kissinger, Analyt. Chem. 29, No. 11, 1702 (1957).

[15] H. Eickenbusch, W. Paulus, E. Gocke, J. F. March, H. Koch u. R. Schöllhorn, Angew. Chem. 99, 1201 (1987).

[16] H. Eickenbusch, W. Paulus, R. Schöllhorn u. R. Schlögl, Mater. Res. Bull. 22 (1987).

[17] I. Haller, M. W. Shafer, R. Figat u. D. B. Goland, Pure and Appl. Chem. im Druck.

[18] M. F. Garbauskas, R. W. Green, R. H. Arendt u. J. S. Kasper, Inorg. Chem. 27, 871 (1988). 